\title{
Integrated Guard Channel Synthesis in AESA based Airborne Surveillance Radar
}

\author{
R Rajesh $^{\mathrm{a}^{*}}$, P V Rao ${ }^{\mathrm{a}}$, Suma Varughese ${ }^{\mathrm{a}}$ \\ ${ }^{a}$ Centre for Airborne Systems, Defence Research and Development Organization (DRDO), Belur, Yemlur Post, \\ Bangalore, India-560037.
}

\begin{abstract}
The guard channel is very effective in eliminating side-lobe returns, both targets and discrete clutter, in airborne radar's. The ideal design criterion for the guard channel is that all the side lobes of the main antenna are covered by the guard pattern. In this paper a novel design approach of generating the guard using the elements of the main Active Electronically Scanned Array (AESA) antenna, thereby eliminating the need for a separate antenna, is presented. The ground clutter has an angle dependent Doppler and clutter discrete that leak in through the side lobes, especially the inter-cardinal elevation side lobes looking at the near range, needs to be eliminated. Towards this the design of the weighting coefficients for the guard to cover all the side lobes of the main array is discussed. Furthermore a digital threshold scheme is proposed to improve the effectiveness of guard channel in eliminating side-lobe returns. The detection loss and blanking probability are characterized for the design. The measurement results confirm that the design objectives are met.
\end{abstract}

Index Terms: Guard Channel, AESA, Phase Spoiling, Blanking Probability, Adaptive threshold, Near Field Measurement.

(C) 2016 Published by MECS Publisher. Selection and/or peer review under responsibility of the Research Association of Modern Education and Computer Science

\section{Introduction}

Airborne surveillance radars are migrating to the Active Electronically Scanned Array (AESA) due to advantages like inertia less beam scanning, beam agility and graceful degradation. This paper considers a long range surveillance radar using the AESA technology. The AESA antenna array of the surveillance radar has slots as radiating elements arranged in a suitable rectangular grid to provide pencil beam in azimuth and fan beam in elevation. Each column is powered by one Receive Multi Module (TRMM). Each TRMM comprises of multiple Transmit - Receive (TR) modules. Such an arrangement provides scanning capability in both azimuth and elevation. For easy maintenance purposes the antenna is manufactured as multiple panels. The

* Corresponding author

E-mail address: rajesh81r@gmail.com 
inter-element spacing of antenna elements is in such a way that it avoids azimuth grating lobes in the scan region of interest. Also, the antenna has 2-D scan capability.

The major challenge in air-borne radars of this class is to provide target detection while competing with clutter returns from the ground. Due to the physical size limitations of the antenna that can be mounted on aircraft, design of ultra-side lobe antennas in such radars is challenging. Hence there is a need of a mechanism to reject both the target returns and discrete clutter that leaks in through the side-lobe. The guard channel provides such a mechanism by comparing the amplitudes of the main and the guard channel. Such a guard channel is also called side lobe blanker (SLB). The guard channel is often synthesised using a separate antenna co-located with the main antenna. In airborne applications such an implementation may not be optimal due to space limitations. The prime design criterion for the AESA based guard channel is that the guard antenna pattern should cover the all side lobes of the main antenna. The design should be robust against amplitude and phase mismatches and also be amenable to implementation using a finite resolution phase shifter.

A practically implementable integrated guard channel design for AESA radars is proposed and the paper makes the following contributions:

- The challenge of matching the noise figure in both the main and side-lobe channel by matching both the paths is addressed by using similar radiating elements and TR modules. This design eliminates the need for separate guard antennas and allows a simple digital threshold scheme that improves the side lobe signal power and clutter rejection capability.

- The paper provides a scheme for design of a robust guard pattern using phase spoiling technique. Such a pattern adaptation improves the SLB coverage keeping the gain reduction due to pattern adaptation within limits.

- The proposed design is characterized in terms of SLB performance like detection loss, probability of detection and probability of side-lobe blanking. It is shown that side-lobe blanking performance of 95\% can be achieved with average detection loss of less than $4 \%$ (across the scan angles).

- The design is validated by Near Field Measurements and the measured results show close proximity to the simulated performance.

The rest of the paper is organised as follows. Section 2 surveys the related work. Section 3 provides the basic model of the guard channel and Section 4 provides the basic design. Section 5 provides the simulation results and Section 6 characterizes the guard performance. Section 7 provides the measurement results and Section 8 concludes the paper.

\section{Related Work}

The SLB was used in early radars to turn off the radar receiver output when an unwanted signal appears in the main lobe. Early work on SLB design is [1]. The characterization of performance of SLB in terms of probability of side-lobe blanking and probability of detection loss in the main channel is provided in [2] [3]. Simpson coined the term Guard channel for SLB and demonstrated its use to reduce ground clutter effects due to side lobes of airborne radar [4]. The guard channel is also effective against low duty cycle jammers and not intended for noise jammers [5]. Several designs for side lobe systems are proposed in [6]. A performance comparison of various side-lobe blanker's is provided in [7]. The work in [3] is extended to correlated Gaussian clutter in [8] and to other interference models in [9]. Several auxiliary SLB channels are described in [10]. A phase only beam spoiling technique is proposed in [11], for design of cosecant squared pattern and beam broadening, for generic arrays.

The optimality of Maisel side lobe blanking [3] is characterized in [12]. The performance analysis of SLB systems in correlated ground clutter and mutual coupling are provided in [13] and [14] respectively. SLB performance for Swirling target is given in [15]. Design of SLB for a phased array based pulse Doppler radar in Doppler domain is given in [16]. Real time aspects of SLB implementation is discussed in [17]. 
None of the above works consider an integrated approach to guard design along with the main antenna. In this paper the flexibility of the active phased array is leveraged in guard design and its performance characterization.

\section{Guard Channel Configuration}

In the AESA radar described in Section I, the guard pattern is synthesised using the 4 elements (of the end columns) of the main antenna. This end column is fed by a TRMM. Due to the usage of one column for guard, the variation in performance of main antenna as compared to the total elements being used only for main channel is marginal and does not affect the performance parameters of the radar. The guard configuration is depicted in Fig. 1:

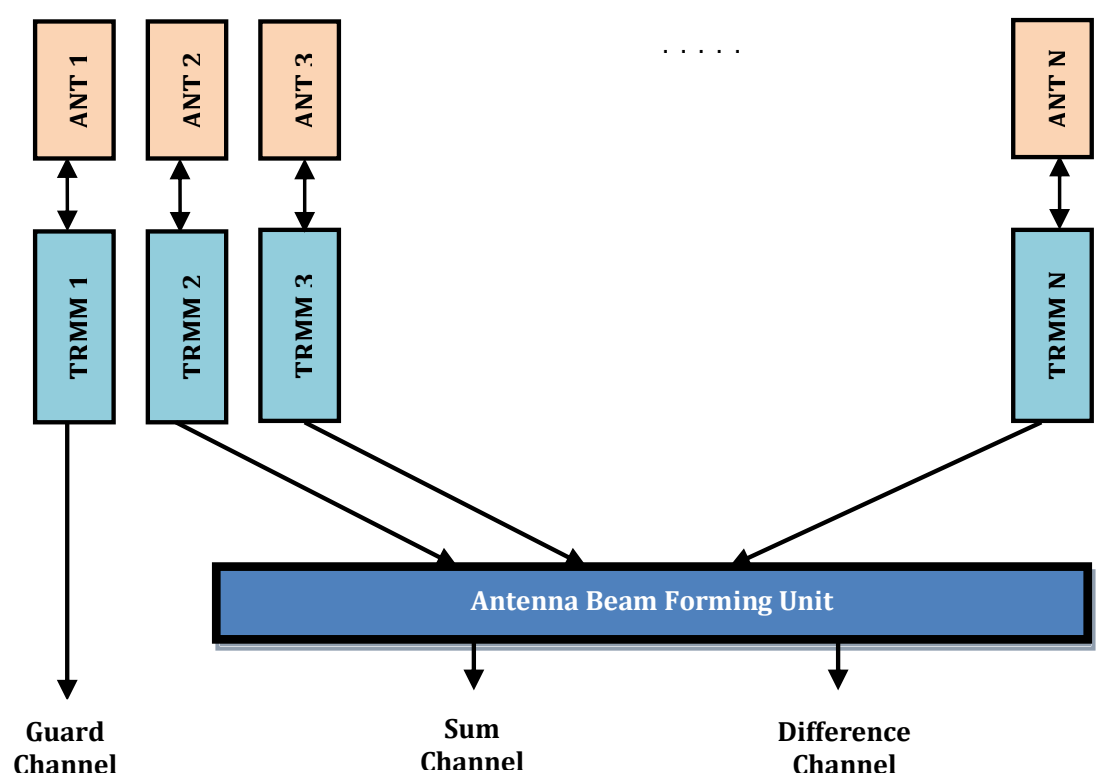

Fig.1. Guard channel configuration

Another advantage of such a design is the noise figure (NF) is matched both the main and the guard channel as the guard sees the same components as the main channel till the amplitude comparison point. Hence the guard design boils down to designing a suitable antenna pattern. Thus, a signal is declared as a side lobe return by suitable comparison of the amplitudes obtained in both the main and guard channel.

\section{Guard Channel Design}

For deriving a suitable design of the guard the main antenna characterises in terms of main lobe and side lobe is analysed. This analysis also takes into account the gain reduction at various off bore sight angles due to electronic scanning. The normalized level diagram in Fig. 2 depicts the parameters of interest. 


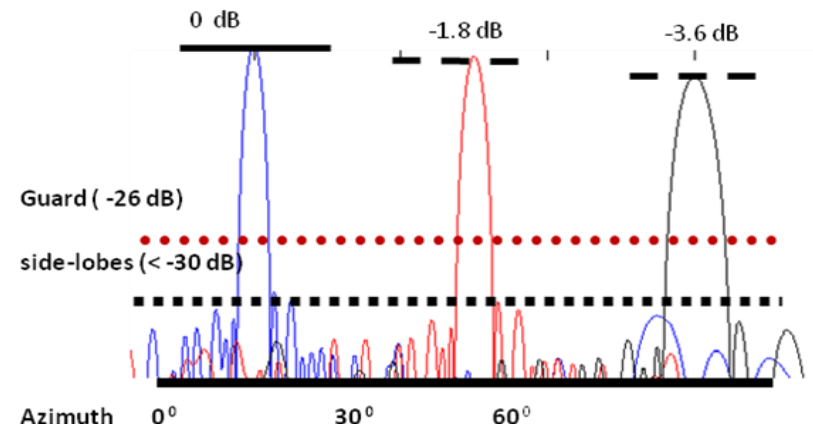

Fig.2. Level diagram for guard design

From Fig. 2 it can be seen that all the side lobes are less than $-30 \mathrm{~dB}$. An un-weighed 4 element guard is at $26 \mathrm{~dB}$ from the main beam peak. Also, the reduction in gain of the main antenna at azimuth scan angles of $45^{\circ}$ and $60^{\circ}$ are indicated. The gain of the guard indicated is without amplitude and phase weighting and thus will have nulls in the pattern. All the gains are normalized with respect to the peak of the main beam. Such a guard pattern with uniform weights is expected to have a broad azimuth beam and an elevation beam with nulls due to the fact that the guard is a column array. The simulated guard channel output in the cardinal planes are given in Figs. 3 and 4.

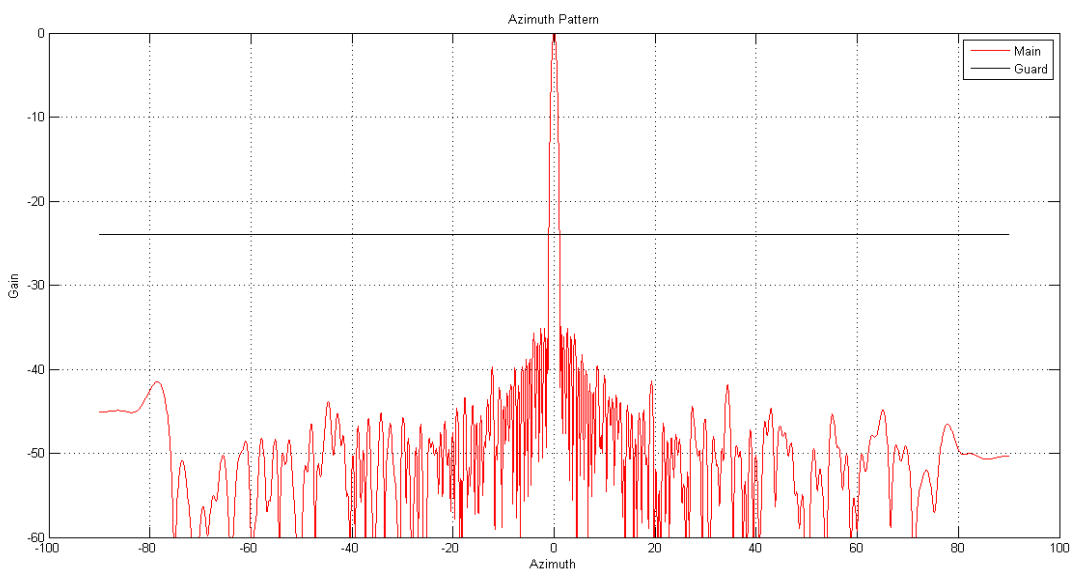

Fig.3. Simulated guard azimuth pattern with uniform weights 


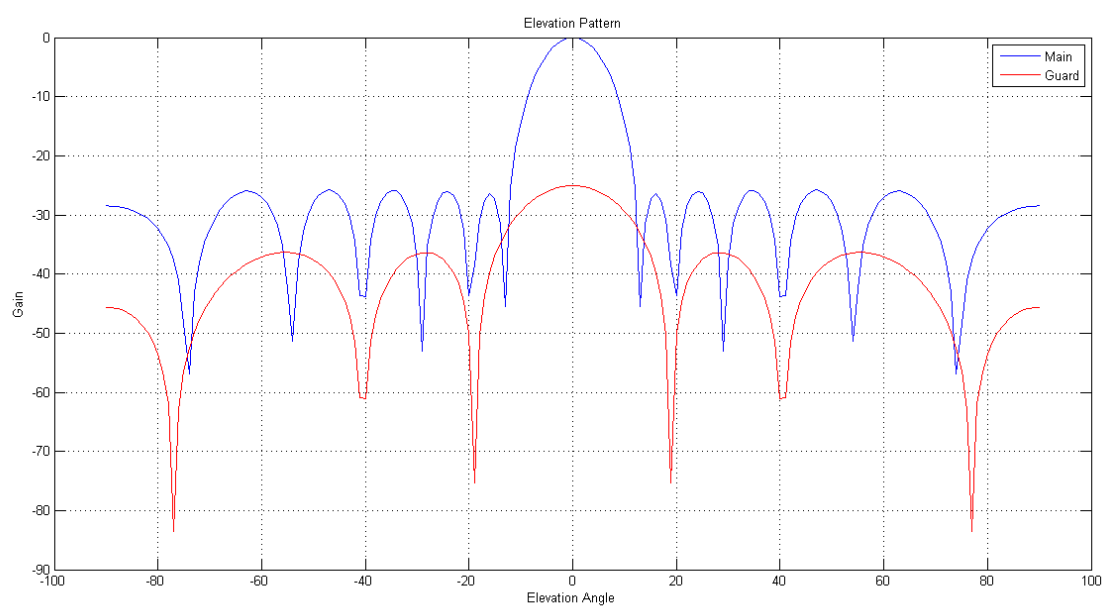

Fig.4. Simulated guard elevation pattern with uniform weights

Such a design suffers from the disadvantage that some of the elevation side lobes are not covered due to the nulls in the guard pattern. Hence suitable weighting coefficients need to be found out so that the guard pattern covers all the side lobes of the main pattern.

Any pattern shaping incurs a loss in gain. Hence, a major consideration in guard pattern shaping apart from that of side-lobe coverage is that the gain reduction due to shaping needs to be kept at the minimum. Hence to meet this objective there is no amplitude taper provided and the pattern shaping needs to be achieved by using phase only taper. Such kinds of phase spoiling techniques are effective for guard design [11].

The following constrained optimization problem is casted for the phase only pattern shaping:

Choose phase vector $[\beta 1, \beta 2, \beta 3, \beta 4]$ in such a way that all the side-lobe are covered by the Guard subject to the constraint that:

The gain reduction due to such a phase vector is within a given tolerance.

Such optimization problems can be re-casted in standard optimization framework by discretization over angular coordinates and can be solved using the techniques provided in [18].

From the set of feasible solution, the optimal solution is arrived by doing a sensitivity analysis with respect to variation in phases and the coefficients that can be implemented in the phase shifter of the TRMM.

The guard channel adaptation using such an approach has the following advantages:

- Main antenna performances are retained and guard is programmable using the same procedure as the main antenna.

- Implementable using the Phase shifters of the TRMMs.

- Robust against phase errors.

- Angle independent design ensures that the same guard coefficients for all main antennas scan angles.

- The main antenna side-lobes are covered.

- The gain loss due in the guard due to pattern adaptation is within specified limits. 


\section{Simulation Results}

The simulation results are provided for the parameter settings provided in Section 4. However the conclusions derived hold in general and can be easily extended for similar designs. The guard is designed using phase only pattern adaptation using the technique given above. The azimuth and elevation pattern are given in the Figs. 5 and 6

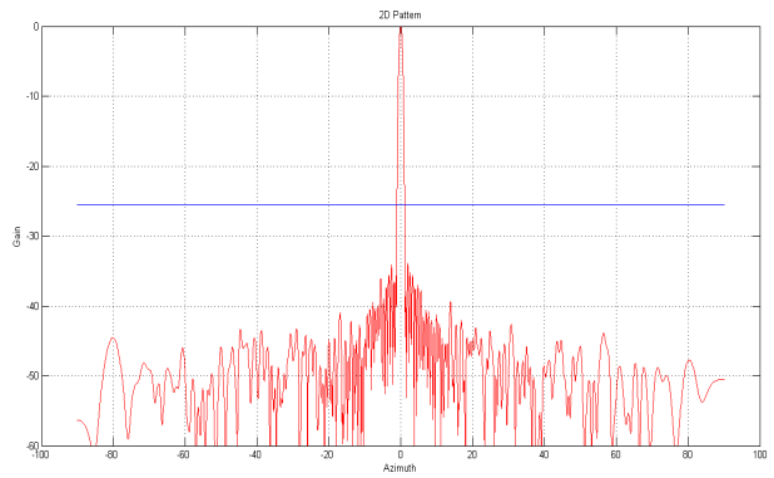

Fig.5. Guard design with phase only weighting (azimuth pattern)

There is a gain loss associated with any weighting. Comparing Fig. 5 and Fig. 3 it can be seen that the gain loss is of the order of $2.5 \mathrm{~dB}$ due to the phase only weighting.

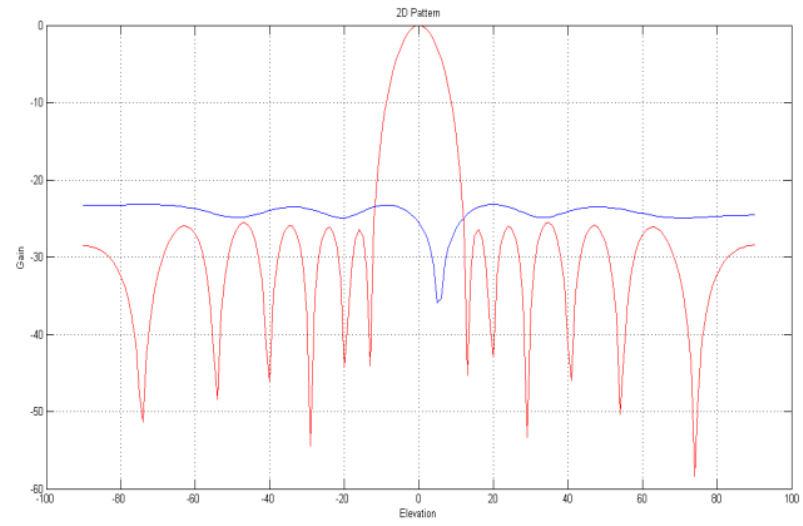

Fig.6. Guard design with phase only weighting (elevation pattern)

From Fig. 6 it can be seen that the phase only weighting re-appropriates the energy in the guard pattern such a way that there is a null formed in the main lobe and all the elevation side lobes are covered. Figure 7 shows the difference between the guard and the main channel plotted in $\mathrm{dB}$ scale. The position of the null is asymmetric so that it is still inside the main beam during look down elevation scanning. Also it can be seen that there is at least $2 \mathrm{~dB}$ difference between the guard and the main antenna side lobes in the cardinal planes. This depicts the worst case and this gap increases in other planes. 


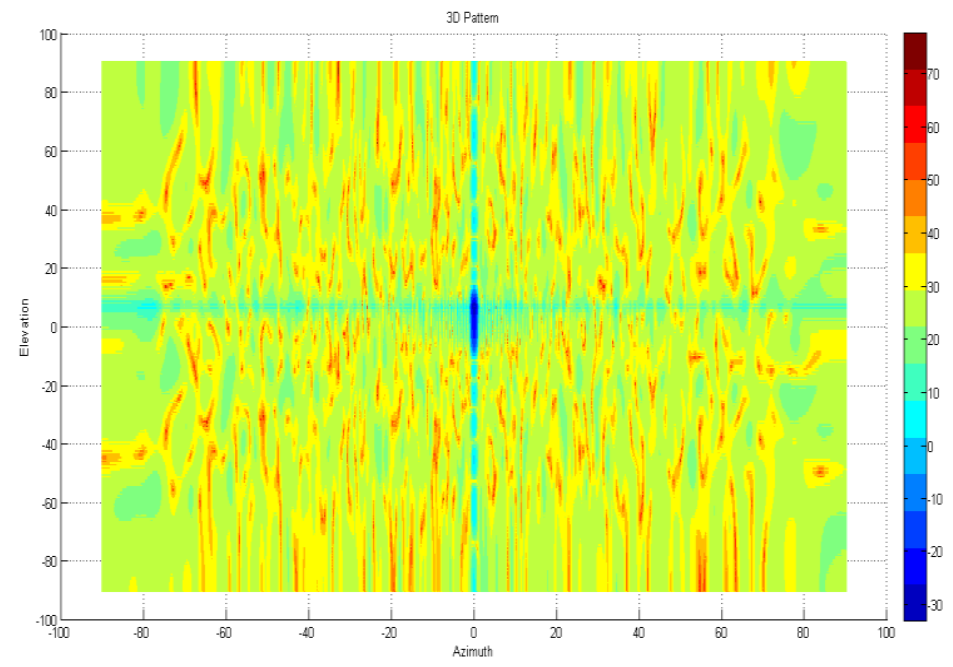

Fig.7. Difference between the guard and the main pattern

From Fig. 7 it can be seen that the guard covers all the side-lobes of the main antenna in the two dimensional pattern except for the main lobe. It can be seen that all the inter-cardinal side lobes are covered with more than $30 \mathrm{~dB}$. This is important in rejecting clutter discrete that leaks in at near ranges.

Such a phase spoiled guard pattern has the following advantages:

- As noticed from Fig. 6, the guard null is inside the main beam and this improves the detection loss under severe clutter and multiple jammers.

- Since the energy is reduced due to the null in the guard, it is re-appropriated suitably to cover the side lobe thus improving blanking performance.

- The antenna pattern remains same in the azimuth except for a gain reduction.

- The gain reduction due to the pattern adaptation is kept at a reasonable minimum.

Hence such an approach to guard design has all the desirable characteristics and amenable to airborne AESA implementations. The main antenna has 2-D scan capability. This is enabled by progressive phase shifts programmed in the phase shifter of the TRMM. The guard has no scanning capability. Hence the phase coefficients are fixed. Figure 8 shows the performance of the guard under an extreme scanned situation of the main antenna. As can be seen from the Fig 8 the performance of the guard is good, that it covers all side lobes, even under extreme scanned conditions. The guard has a broad beam width in the azimuth direction which is unaffected by the pattern shaping in elevation. Thus such a guard design with phase spoiling based pattern adaptation covers all the main antenna side lobes under 2-D scanned conditions. 


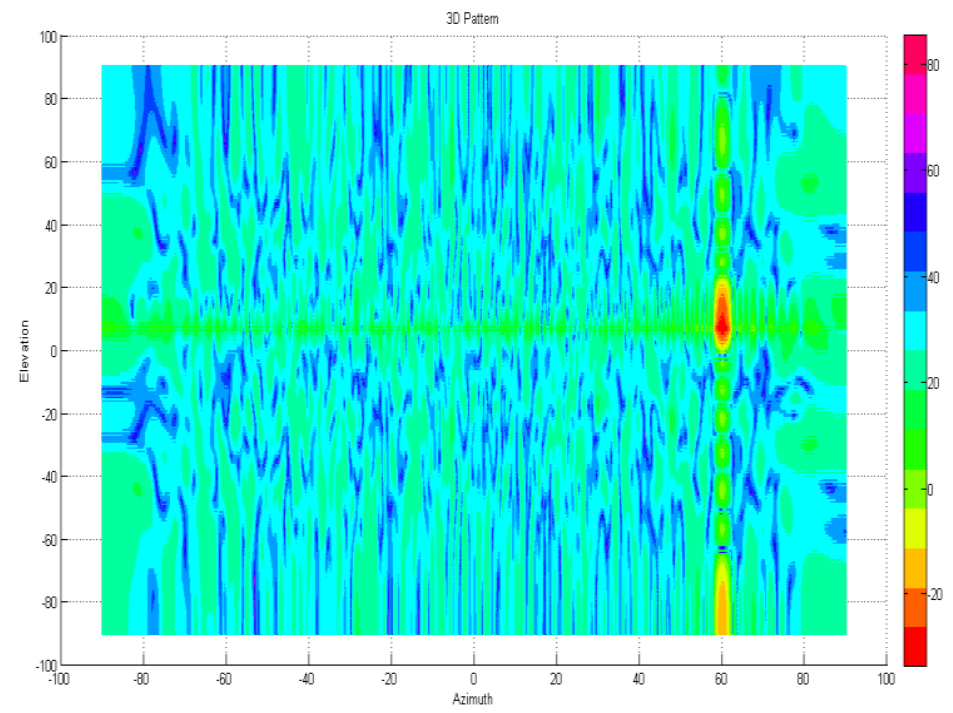

Fig.8. 3 -D difference between the guard and the main pattern $\left(60^{\circ}, 10^{0}\right)$

\section{Digital Threshold Scheme}

In this section the basic technique through which the guard improves the main antenna performance, under jamming and clutter scenarios, is explained. For each main channel return, Signal to noise ratio (SNR_main) is calculated. It is compared with the corresponding guard channel return SNR_guard. As the main and the guard paths are designed in such a way that the noise figure of both the channels is similar, a straight forward comparison is possible. The comparison is performed at the logarithmic scale. Hence a signal return is declared as from the main beam if

$$
S N R \_ \text {main-SNR_guard }>\delta
$$

Where $\delta$ is a suitable threshold so that for a fixed probability of false alarm (return from the side-lobe is declared as from the main lobe) the probability of detection (return from main-lobe is not missed) is maximized. By suitable adaptation of $\delta$ at the amplitude comparison point, the effectiveness of the guard technique and thereby the side lobe blanking (SLB) ability can be improved.

The important parameter in characterizing guard channel performance is the probability of blanking $\left(\mathrm{P}_{\mathrm{B}}\right)$ and probability of detection $\left(\mathrm{P}_{\mathrm{D}}\right)$. Probability of blanking is the probability that a side lobe return is blanked by the guard. Probability of detection is the probability that a main channel return is not masked by the guard. This can be also expressed in terms of detection loss which is $1-\mathrm{P}_{\mathrm{D}}$.

The characterization of the various probabilities with guard is adapted from [3]. The probabilities are plotted for various threshold levels $\delta$ in Fig. 9 under the following assumptions:

- Both the side lobe and guard are at the same level.

- The side-lobes are flat. 


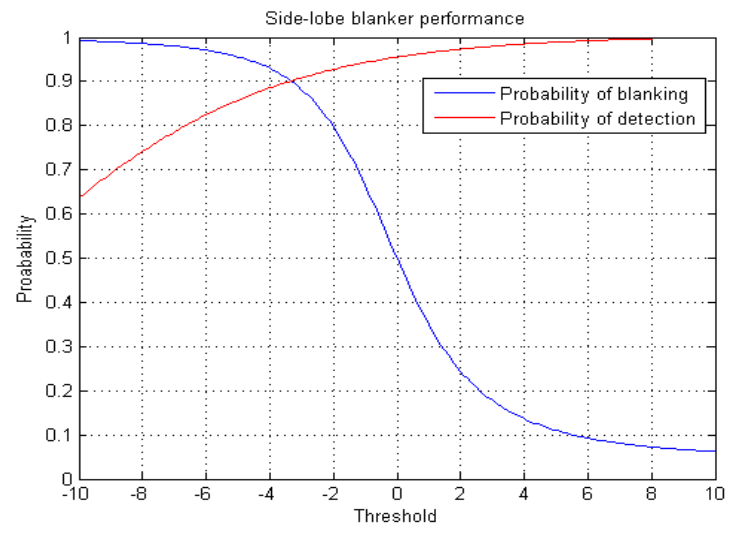

Fig.9. Probability of side-lobe blanking and probability of detection

Hence from Fig. 9, it can be see that an optimum point exist that provides good $\mathrm{P}_{\mathrm{B}}$ and $\mathrm{P}_{\mathrm{D}}$ (e.g., More than $90 \% \mathrm{P}_{\mathrm{B}}$ and $\mathrm{P}_{\mathrm{D}}$ are achieved at $\delta=-3.5$ ). In the proposed AESA, the gains of the guard and the main antenna are such that the guard channel covers all the side lobes (Fig. 7). The logarithmic difference between the guard channel gain and the side lobe gain (of the main antenna) is denoted as gain ratio. The gain ratio is a function of scan angle due to the fact that there is a scan loss due to the reduction in effective aperture. The gain ratio is 8 $\mathrm{dB}$ at zero degree scan and around $1 \mathrm{~dB}$ at 60 degree scan. Also, the side lobe control is through Taylor pattern; hence the side lobes are not constant and have a roll of rate. A suitable compensating factor can be used to account for the performance of proposed AESA which has side lobes with defined roll of rate. For the Taylor pattern the worst case compensating factor assumed is around $30 \%$ (i.e., around $70 \%$ of the flat side lobe case). For comparison purposes a compensating factor of $50 \%$ is also considered.

Next the detection loss is characterized in Fig. 10 for various scan angles for the proposed AESA for the requirement of $95 \%$ blanking probability. The detection loss is characterized as a function of gain ratio (equivalently scan angles). The gain ratio decreases from around $8 \mathrm{~dB}$ to $0.5 \mathrm{~dB}$ as the antenna is scanned from $0^{0}$ to $60^{\circ}$. This reduction of gain is due to the combined effect of gain reduction and side lobe increase due to scanning.

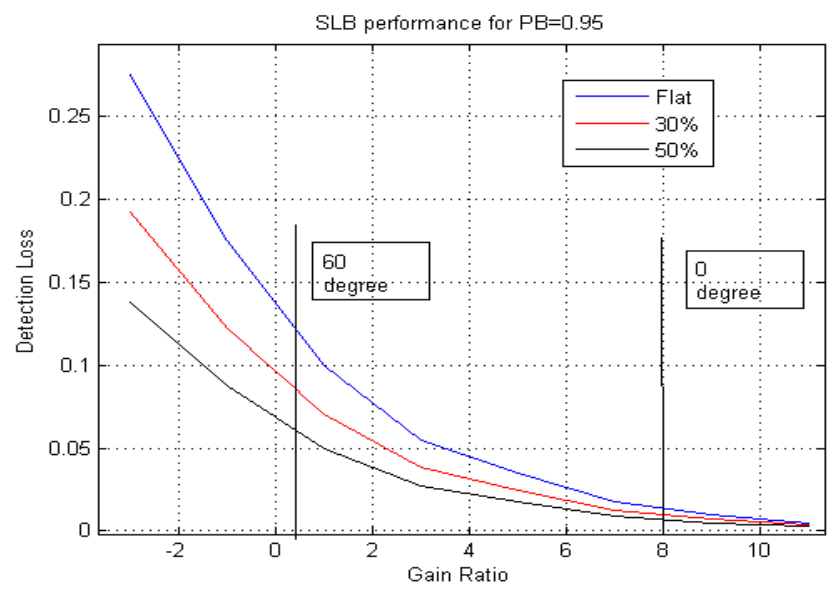

Fig.10. Characterization of detection loss 
From Fig.10 we can derive the following conclusions:

- The worst case detection loss under extreme angles is less than $8 \%$.

- The average detection loss is less than $4 \%$.

- There is a need to use an adaptive comparison threshold $\delta$ with scan angles to improve the performance.

In Fig. 11 we plot the adaptive threshold $\delta$ that needs to be chosen corresponding to various scan angles (gain ratios). Such an adaptive threshold chooses an optimum operating point in the curve minimizing the detection loss for a chosen blanking probability. The threshold also ensures that $P_{B}$ and $P_{D}$ are invariant to scan angles and provides uniform performance across various directions.

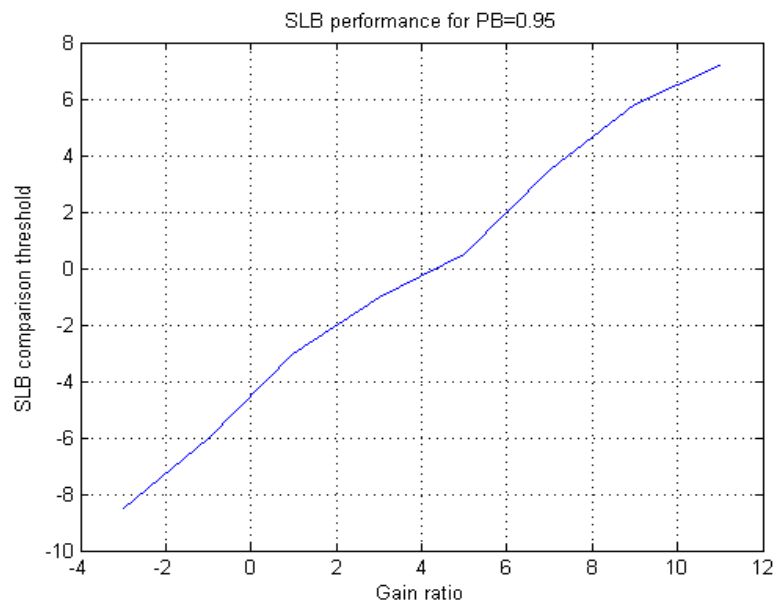

Fig.11. Adaptive threshold

The adaptive threshold can be easily programmed for various scan angles of the array. From Fig. 11 it can be seen that digital threshold for comparison needs to be lowered as we increase the scan angle (equivalently decrease the gain ratio). The threshold is almost linear with the gain ratio. This variable comparison threshold ensures that it provides a good blanking probability at minimal detection loss.

\section{Measurement Results}

The AESA array has been tested in the Near-Field Measurement (NFM) setup. The NFM has the near field scan area coverage for about $24 \mathrm{~m} \times 8 \mathrm{~m}$. The high gain and larger aperture antenna or antenna array are tested at the facility. The chamber permits to study the antennas which are for the frequency higher than $1 \mathrm{GHz}$. The chamber also fitted with high power absorbers to accommodate the evaluation of array under transmit full power mode. The test facility has the capability of defining the dimension of the sample space, grid size for the sample, multi frequency selection and multi scan angle settings. The measurement is carried out for the Receive mode of the AESA array.

Figure 12 shows the guard pattern overlaid on the far field pattern of the main AESA array in the receive mode. The plots are also shown for the scan angle patterns overlaid with guard pattern. The individual zero normalized patterns obtained from the measurement set up are combined in Fig 12. The plots describe the complete coverage of the side lobes with the guard pattern as it is supposed to be. Figure 13 shows the coverage of the guard pattern with the elevation pattern of the AESA array. The plots also describe good coverage of the side lobe levels of the main pattern by the guard pattern. The mismatch in the extreme angular 
region is imposed due to the limitation of scan area coverage in the near field measurements. This is the inherent limitation of the Near field measurements the behaviour at the elevation could be attributed to the measurement limitations.

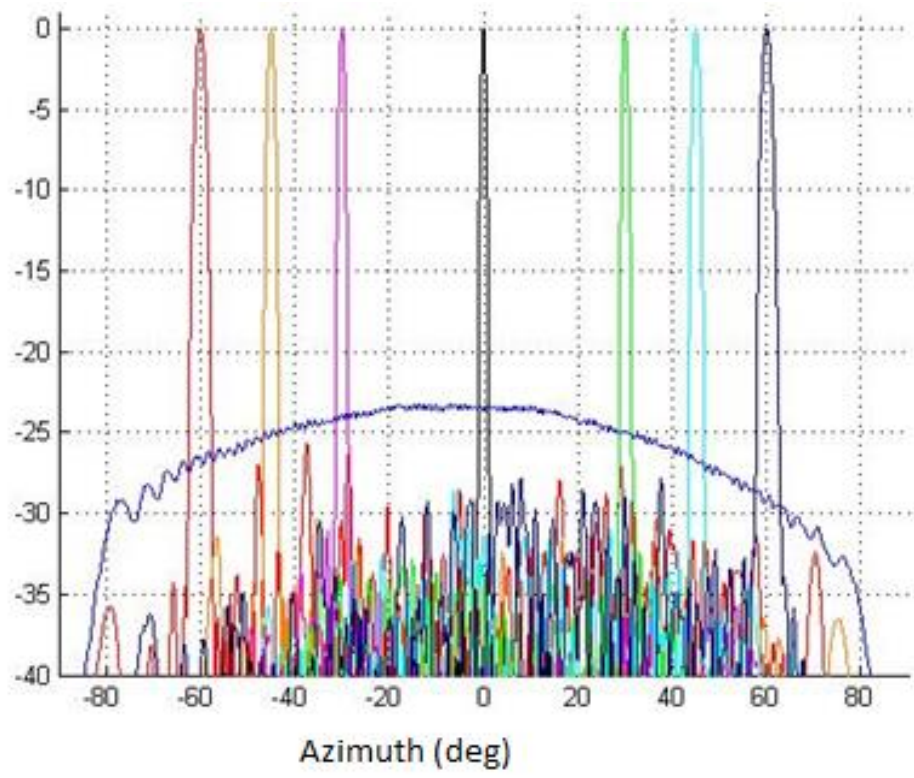

Fig.12. Guard pattern overlaid on Azimuth Scan angle patterns

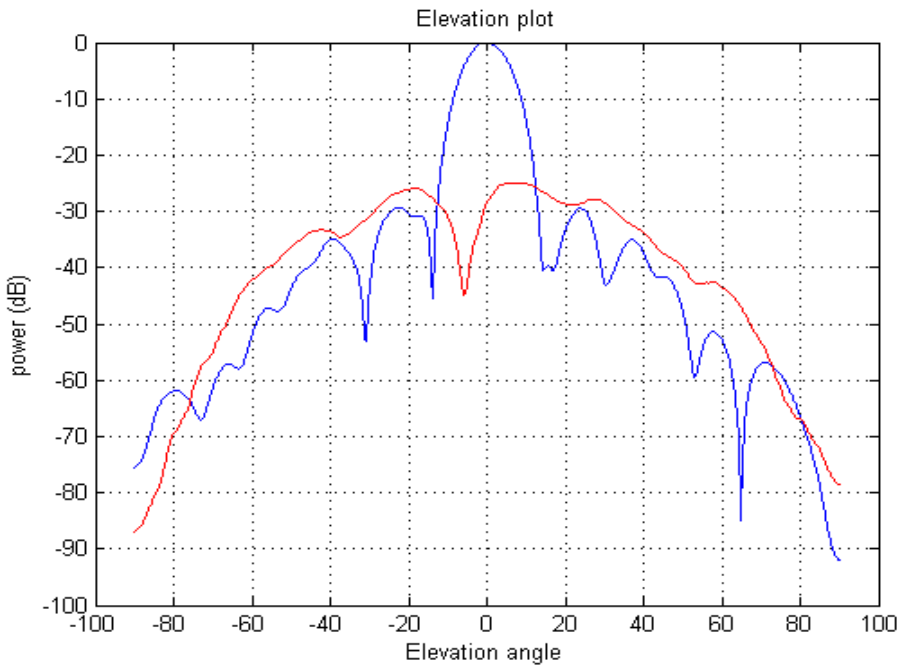

Fig.13. Guard pattern overlaid on Elevation far field pattern

\section{Conclusions}

An end to end design technique for integrated guard channel synthesis in AESA based radars has been 
provided. In the proposed design, the Noise figures in the main and guard channel has been matched. The guard pattern has been adapted to cover the main beam side lobes by phase spoiling technique. The proposed design is seen to minimize gain losses of the guard by providing a null in the main beam and increased gain in the side-lobe direction. From the performance analysis, it has been found that the guard channel provides a blanking probability of $95 \%$ with the average detection loss of less than $4 \%$. Further by using an adaptive digital threshold scheme the effectiveness of the guard have been improved at higher scan angles. The simulation results and the design have been seen to match closely with the measurements obtained from the near field measurement facility.

\section{References}

[1] Aranciba P. O, Side-lobe blanking system design and demonstration, Microwave journal Vol 21, No3, March 1978.

[2] Johnster S L (ed), Radar electronic counter counter measures, Artech house, 1979.

[3] Maisel L, Performance of side-lobe blanking systems, IEEE Trans Aerospace and Electronic systems Vol 4, No 2, March 1968.

[4] Simpson G . W, Introduction to airborne radar, Huges aircraft co., 1983.

[5] W C Morchin, Airborne early warning radar, Artech house, 1990.

[6] Harvey D. H and Wood T. L, Designs for side-lobe blanking systems, Proceedings of IEEE international radar conference, April 1980.

[7] O' Sullivan M R, A comparison of sidelobe blanking systems, Proceedings of IEEE international radar conference, April 1987.

[8] Farina A and Gini F, Interference blanking probabilities for SLB in correlated Gaussian clutter and noise, IEEE Trans on signal processing, Vol 48, No 5, May 2000.

[9] Farina A and Gini F, Calculation of blanking probability for the sidelobe blanking for two interference statistical modes, IEEE Signal processing letters, Vol 5, No 4, April 1998.

[10] Johnson M A and SC Storer, ECCM from the radar designers viewpoint, IEEE Electronics-76, May 1976.

[11] R Kinsey, Phased array beam spoiling technique, IEEE Trans. Antennas and Propagation, 1997.

[12] O Coksun, C. Canden, On Optimality of Maisel side lobe blanking, $23^{\text {rd }}$ Signal processing and communication applications conference, pp 585-591, 2015.

[13] Farina A and Gini F, Design of SLB systems in the presence of correlated ground clutter, IEE proceedings on Radar, Sonar and Navigation, Issue 4, vol 147, 2000.

[14] A De Maio, A. Farina, M Fiorini, A. Morini, Performance analysis of side lobe blanking systems in presence of mutual coupling, IET international conference on radar systems, pp 1-5, 2007.

[15] O' Sullivan M R, Side lobe blanking system performance for Swirling target, International Conference on Radar, April 1989.

[16] F. Mingyi, G. Jianjun, Q. Weil, W, Manqing, Side lobe blanking in Doppler domain for an Airborne side looking phased array PD radar, CIE International Conference on Radar, 20016.

[17] B. Magaz, M. L. Bencheku, H. Hamadouche, A. Belouchrani, Design and real time implementation of a novel combined CA-CFAR/SLB system on TMS 3200C67x processor, International Radar Symposium, pp 1-4, 2006.

[18] S Boyd and L. Vandenberghe, Convex optimization, Cambridge university Press, 2008. 


\section{Authors' Profiles}

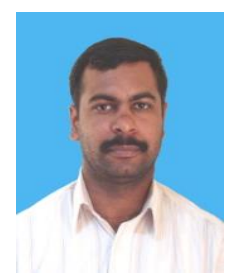

R Rajesh received his B-Tech in ECE from National Institute of Technology, Calicut, India in 2002 and $\mathrm{PhD}$ from Electrical Communication Engineering department, Indian Institute of Science, Bangalore, India in 2009. Since December 2002, he is working as a Scientist with Centre for Airborne Systems, DRDO, Bangalore. His areas of interest include Information Theory, Wireless Communication, Radar Systems and System Engineering. He is a recipient of Laboratory Scientist of the year award in 2006, DRDO Young Scientist award in 2008 and Technology group award in 2013.

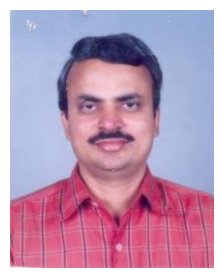

P V Rao received his MSc (Engg) on adaptive arrays in 1993 and $\mathrm{PhD}$ in computational electromagnetics in 2002 from Indian Institute of Science, Bangalore. Since 2005 he is working as a Scientist with Centre for Airborne Systems, DRDO. His areas of interest include Antenna, Antenna Arrays, Adaptive Arrays, Phased Array, Active Antenna Array, Computational Electromagnetic, RCS analysis and Mounted Antenna Analysis. He is a recipient of Laboratory scientist of the year award in 2012 and IETE M N Saha best paper award in 2013.

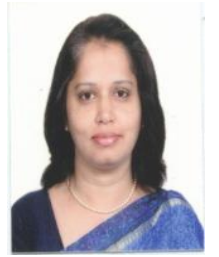

Suma Varughese received her MSc (Comp. Sci.) in 1988 and MS (Engg) on Antenna measurement techniques from Institute of Science, Bangalore. She joined LRDE, DRDO in 1989. She moved to CABS, DRDO, Bangalroe to join a prestigious airborne programme in 2004. She currently is an outstanding scientist at CABS heading various airborne system projects. Her areas of interest include active array antennas, antenna measurement techniques, muti sensor data fusion and system engineering. She is a receipient of IETE J C Bose gold medal for best paper in 1993, NRDC national award for PNFM software in 1995, IETE N M Saha best paper award in 2013 and DRDO Agni award in 2013.

How to cite this paper: R Rajesh, P V Rao, Suma Varughese,"Integrated Guard Channel Synthesis in AESA based Airborne Surveillance Radar", International Journal of Wireless and Microwave Technologies(IJWMT), Vol.6, No.6, pp.1-13, 2016.DOI: 10.5815/ijwmt.2016.06.01 